\title{
Experiencias de trabajos en educación
}

\author{
Mauro Pérez Morales \\ mperez@uncp.edu.pe \\ https://orcid.org/0000-0001-6960-7212
}

\section{Resumen}

El presente estudio de investigación se circunscribe en el contexto del desarrollo del MINEDU, como son las actividades de supervisión y monitoreo del Diplomado en Gestión Escolar y Segunda Especialidad en Gestión Escolar con Liderazgo Pedagógico, dirigido a Directores y Sub Directores del ámbito peruano a partir del año 2016, en este caso solo me limitaré a la región Huancavelica. En efecto es necesario precisar describir las características del Diplomado, como es desarrollar aspectos trascendentales referentes a gestión escolar, todo esto con la finalidad de mejorar los niveles educativos de los Directores de las diferentes instituciones educativas, quienes logran cambiar en sus conocimientos, comportamiento y actitudes que en lo futuro contribuyen positivamente en la formación de los diferentes directores, sub directores en el marco del buen desempeño directivo, por lo que si considera importante mejorar el liderazgo pedagógico del director, como un pilar fundamental para la mejorar los diferentes procesos de aprendizaje, en este contexto fueron considerados los objetivos y acciones de las IE en el aspecto pedagógico de las instituciones primarias y secundarias del contexto territorial del país. Los mismos que fueron desarrolladas por el Ministerio de Educación, que en este caso específico de la región Huancavelica, enmarcado dentro del contexto de la política del ministerio de educación - MINEDU, que responde a realidades muy complejas y contextos diversos.

Palabras clave: Diplomado, curriculum, management, school and leadership.

\begin{abstract}
This research study is circumscribed in the context of the development of the MINEDU, such as the supervision and monitoring activities of the Diploma in School Management and Second Specialty in School Management with Pedagogical Leadership, aimed at Directors and Deputy Directors from the Peruvian territory, in this case I will only limit myself to the Huancavelica region. In fact, it is necessary to specify the characteristics of the Diploma, such as developing transcendental aspects related to school management, all with the purpose of improving the educational levels of the Directors of the different educational institutions, who manage to change their knowledge, behavior and attitudes that in the future contribute positively to the training of the different directors, assistant directors within the framework of good management performance, so if it is considered important to improve the director's pedagogical leadership, as a fundamental pillar to improve the different learning processes, In this context, the objectives and actions of the EIs were considered in the pedagogical aspect of the primary and secondary institutions of the territorial context of the country. The same that were developed by the Ministry of Education, which in this specific case of the Huancavelica region, framed within the context of the policy of the Ministry of Education - MINEDU, which responds to very complex realities and diverse contexts.
\end{abstract}

Keywords: Diploma, curriculum, management, school and leadership. 


\section{Introducción}

El presente artículo de investigación constituye la primera parte de investigación formulada acerca de la supervisión y monitoreo del programa de Diplomado en gestión escolar y segunda especialidad en gestión escolar con liderazgo pedagógico, que se impartieron a los Directores y Sub Directores del ámbito del territorio peruano y específicamente en la jurisdicción de la región Huancavelica, que es materia de la presente investigación explorativa.

En efecto el "Diplomado en Gestión Escolar y Segunda Especialidad en Gestión Escolar con mención en Liderazgo Pedagógico". Forma parte de la investigación a nivel nacional, así en la presente investigación se plasmó el método descriptivo, complementando de este modo con contenido de Gestión escolar emanada del Estado, plasmada con contenidos a la educación secundaria, complementada como aspectos relacionados con la educación por competencias.

Por otra parte, vale precisar el trabajo emprendido e histórico por el Estado peruano, el emprendimiento del diplomado, realizada con una ceremonia de inauguración del Diplomado en Gestión Escolar y Segunda Especialidad en Gestión Escolar con mención en Liderazgo Pedagógico, junio 2016 dirigido a más de 15000 directores y sub directores de las instituciones educativas públicas de Educación Básica Regular (EBR), Educación Básica Especial (EBE), Educación Básica Alternativa (EBA) y educación Técnico Productiva (ETP) del Perú, que la responsabilidad estuvo a cargo de prestigiosas instituciones, Universidades del Perú.

Describir cuales son los impactos generados por efectos del Diplomado en gestión escolar y segunda especialidad en gestión escolar con liderazgo pedagógico", impulsados por el Ministerio de Educación (Minedu), dirigidos a los directores y sub directores del país, en este caso específico y en particular de la región Huancavelica.

En relación a la investigación es descriptiva, por cuanto los impactos del Diplomado, si contribuyó positivamente en la formación de los directores, sub directores en el marco del buen desempeño directivo que reconoce el liderazgo pedagógico del director o directora de la IE, como pilar fundamental para la mejora de los aprendizajes, centrando los objetivos y acciones de la IE en lo pedagógico, a nivel de las instituciones primarias y secundarias del contexto territorial.

La investigación es de carácter descriptivo, que describe los detalles y los resultados, de la aplicación de los diferentes instrumentos de recojo de información de la visita inicial del Módulo 0 a las diferentes directores y sub directores, de las sedes que fueron consideradas a instituciones de nivel secundario, superior del ámbito de la provincia de Huancavelica, Castrovirreyna y Angaraes respectivamente, con el propósito de realizar el seguimiento a los docentes directivos participantes.

Referente a la aplicación de los diferentes instrumentos de recojo de información de la visita inicial del Módulo 0, del "Diplomado de Gestión Escolar y Segunda Especialidad en Gestión Escolar con Liderazgo Pedagógico" en inicio con un proceso riguroso de evaluación, luego la coordinación respectiva realizada con funcionarios de la UGEL, DREH y funcionarios del MINEDU, se realizó la implementación, capacitación de modo descentralizado, como es en este caso en la ciudad de Huancavelica. Consecuentemente se realizaron las diferentes visitas y monitoreo de los trabajos de aplicación de los diferentes instrumentos en sus respectivas sedes, sub sedes del Diplomado en Gestión Escolar realizados a nivel de las provincias de Huancavelica, Angaraes, Castrovirreyna. 
Es esta circunstancia es necesario mencionar que en las diferentes instituciones educativas del nivel primario y secundario, hemos percibido realidades diversas, con una realidad marcada cada vez influenciada por la globalización, a su vez por la tecnología, la modernidad y una economía de mercado que permite mirar hacia afuera lo externo.

Consideramos de vital importancia el desarrollo de las actividades del diplomado complementadas por las diversas actividades de supervisión y monitoreo durante el Diplomado en Gestión Escolar y Segunda Especialidad en Gestión Escolar con Liderazgo Pedagógico, que se impartieron a los Directores y Sub Directores del ámbito jurisdiccional de la Región Huancavelica, donde vale precisar referente a las diferentes vías, aquellas ciudades, centros poblados que se encuentran vinculadas a los principales ejes viales, los que facilitaron el acceso para el desarrollo y la realización de los trabajos de campo, donde en el primer caso se suele observar la ubicación de ejes viales que en algunos casos son trochas.

Es probable que se ha percibido y escuchado respecto al diplomado que se encuentra dentro del marco del buen desempeño Directivo (Minedu. 2014), que constituye una herramienta del líder pedagógico, con sustento de la literatura sobre gestión escolar, mejora y cambio educativo, dirigido a instituciones eficaces, calidad educativa, aprendizaje organizativo, donde el diplomado va dirigido a directores y subdirectores de IE públicas de los diferentes niveles todo este proceso complementado en función a la formación profesional, respaldado por las diversas manifestaciones socioculturales, relacionadas entre sí que cotidianamente encontramos, sin embargo no son valorados su utilidad y contribución en las actividades del Diplomado en Gestión Escolar y Segunda Especialidad en Gestión Escolar con Liderazgo Pedagógico, que se impartieron a los Directores y Sub Directores del ámbito jurisdiccional de la región Huancavelica. Que, dentro de su estructura contenidos, consideran aspectos que tienen relación, como:

Participación de directores y subdirectores en el diplomado en gestión escolar y segunda especialidad en gestión escolar con liderazgo pedagógico.

Características para la participación de directores y subdirectores en el diplomado en gestión escolar y segunda especialidad en gestión escolar con liderazgo pedagógico.

Guía para la participación de directores y subdirectores en el diplomado en gestión escolar y segunda especialidad en gestión escolar con liderazgo pedagógico.

Para el diplomado: Guía para la participación de directores y subdirectores en el diplomado en gestión escolar y segunda especialidad en gestión escolar con liderazgo pedagógico 5.

Guía para la participación de directores y subdirectores en el diplomado en gestión escolar y segunda especialidad en gestión escolar con liderazgo pedagógico 66 .

\section{Aspectos teoricos}

En el Perú, se viene implementando diversos programas, así el Minedu, viene poniendo en práctica acciones orientadas a directores y subdirectores de instituciones educativas públicas de todas las regiones del país, fueron capacitados por el Ministerio de Educación (Minedu), en particular los directores y sub directores de la región Huancavelica, dentro de ellos Angaraes, Castrovirreyna y Huancavelica respectivamente, con el propósito de que desarrollen competencias y desempeños que les permitan realizar gestiones en beneficio de sus instituciones educativas, las evaluaciones desarrolladas por las entidades formadoras. 
Así, el currículo educativo es una herramienta didáctica importantes de los profesores que incluye los criterios, los planes de estudios, la metodología, los programas y todo relacionada a los procesos que servirán en lo futuro para proporcionar a los estudiantes una formación integral y completa a nivel superior, (Cabrera 2006).

...diferentes experiencias fueron aplicadas en distintos momentos y todas tuvieron el común denominador de apostar y focalizar nuestra intervención con los jóvenes, Nuestras intervenciones incluyeron el fomento de la participación ciudadana a través de la consolidación de espacios administrados por jóvenes, el impulso del emprendimiento a través de herramientas...

Sin embargo, los contenidos del currículo educativo oficial, se circunscribe en la estructura del gobierno nacional, lo cual es implementada a través de los ministerios, mediante un conjunto de normas, reglamentos y afines, para el cumplimiento por las diferentes instituciones superiores, plasmados en los procesos de enseñanza - aprendizaje en cada institución del territorio peruano. Actividades de supervisión y monitoreo durante el Diplomado.

Así los estudiantes Actividades de supervisión y monitoreo durante el Diplomado en Gestión Escolar y Segunda Especialidad en Gestión Escolar con Liderazgo Pedagógico, que se impartieron a los directores y Sub directores del ámbito jurisdiccional de la Región Huancavelica. En la realización de actividades de formación a los directores y sub directores, se observa la relación de las prácticas de liderazgo, en el marco del buen desempeño del directivo, las competencias del Diplomado y Segunda Especialidad, donde es necesario tomar en cuenta el modo de vida de las personas como parte de una cultura, organización, donde permite interpretar la cotidianidad del ser humano, lo que hace y dice, al respecto (Psyma, 2015) menciona:

"La etnografía es uno de los métodos más relevantes que se utilizan en la investigación con enfoque cualitativa. Es un método de investigación por el que se aprende el modo de vida de una unidad social concreta, pudiendo ser ésta una familia, una clase social o una escuela. Permite interpretar el día a día del consumidor desde lo que hace y no sólo por lo que dice que hace, enfocados a comprender los códigos culturales que rodean a una marca o a un producto especifico".

Se debe tener en cuenta los contenidos del Diplomado, contenido como son el marco del buen desempeño del director, orientados a promover y liderar una comunidad de aprendizaje con los docentes de su institución educativa, basada en la colaboración mutua, autoevaluación profesional y formación continua, orientada a mejorar la práctica pedagógica y asegurar logros de aprendizaje ayuda de un guía experto en el tema. El estudiante cinco, responde a la misma pregunta, para un antropólogo, el trabajo de campo se entiende como el instrumento para aplicar todo lo aprendido en clases, todo lo teórico llevarlo a la práctica, Bourdieu, (1970) menciona.

...han explorado. Dos dimensiones complementarias: el funcionamiento del sistema escolar y las actitudes de las distintas clases sociales en relación a la cultura. Cada uno de ellos desbrozó una parcela, aportó una demostración... no hay complot de las élites ni alienación completa de los dominados, ni máquina infernal al servicio de los intereses de la burguesía. Hay, más bien, un sistema que va transformándose precisamente para 
perpetuar los privilegios de clase, con una lógica que puede desentrañarse a condición de no adjudicarla al engaño deliberado de individuos o grupos...

A partir de una lectura crítica de Bourdieu, pedagogos, docentes, investigadores examinan los métodos pedagógicos, los criterios de ingreso y de evaluación, el valor económico y simbólico de los títulos, el registro de lengua en que se transmiten mediante los contenidos, el desempeño de los docentes y las trayectorias de los estudiantes, tomando en cuenta aprendizajes en entornos extraescolares, como la familia y los espacios donde se imparten una educación de corte nacional.

El "Diplomado en Gestión Escolar y Segunda Especialidad en Gestión Escolar con mención en Liderazgo Pedagógico", empleando métodos y técnicas de investigación adecuada y por otra parte de los estudiantes aprovechada para el relajo caracterizada por la irresponsabilidad y el conformismo que sirvió como comodín para obtener notas aprobatorias esto para algunos estudiantes, contribuidos por los directores y subdirectores de instituciones educativas públicas de todas las regiones del país fueron capacitados por el Ministerio de Educación (Minedu), en particular los directores y sub directores de la región Huancavelica, específicamente en las provincias de Angaraes, Castrovirreyna y Huancavelica respectivamente, con el propósito de que desarrollen competencias y desempeños que les permitan realizar gestiones en beneficio de sus instituciones educativas.

En esta circunstancia, es necesario la valoración, contribuciones y alcances que adquieren los estudiantes del nivel superior, como contar con herramientas necesarias para desenvolverse en la universidad, en su entorno y la sociedad. Atender a la diversidad de cada estudiante superior, prestando atención, apoyo para el desarrollo de aptitudes y cualidades individuales en el proceso de formación profesional. Actividades de supervisión y monitoreo durante el Diplomado en Gestión Escolar y Segunda Especialidad en Gestión Escolar con Liderazgo Pedagógico, estas actividades orientadas a los Directores y Sub Directores del ámbito jurisdiccional de la región Huancavelica.

\section{Resultados}

Según resultados preliminares de la presente investigación es necesario precisar, la importancia que tiene el Actividades de supervisión y monitoreo "Diplomado en Gestión Escolar y Segunda Especialidad en Gestión Escolar con mención en Liderazgo Pedagógico". Director, orientados a promover y liderar una comunidad de aprendizaje con los docentes de su institución educativa, basada en a colaboración mutua, autoevaluación profesional y formación continua, orientada a mejorar la práctica pedagógica y asegurar logros de aprendizaje durante el Diplomado en Gestión Escolar y Segunda Especialidad en Gestión Escolar con Liderazgo Pedagógico, que se impartieron a los directores y sub directores del ámbito jurisdiccional de la Región Huancavelica.

El Minedu, en cumplimiento a sus fines y propósitos educativos a fin de alcanzar objetivos como es la mejora de la calidad educativa, donde funcionarios, trabajadores del sector educación tienen a diferencia del trabajo de docentes en los diferentes sectores rurales que en algunos casos tienen obstáculos, dificultades y barreras que aún son invisibles seguido por 
los contenidos del curriculum oficial y contenidos del curriculum oculto que se complementan y contribuyen a la formación profesional de los estudiantes, docentes de los diferentes niveles del ámbito jurisdiccional, donde complementariamente se desarrolló un módulo para el tratamiento del trabajo académico en el que el directivo presentó una propuesta de mejora para su escuela con fines de titulación, más de 13,536 directores y subdirectores que concluyeron la formación en el Diplomado en Gestión Escolar.

De todos ellos, 6,323 participantes culminaron la Segunda Especialidad en Gestión Escolar con Liderazgo Pedagógico. Un segundo grupo de 7,213 cursaron esta segunda especialidad entre abril y diciembre. Cabe señalar que las autoridades educativas que participaron en estas capacitaciones, realizadas a un costo aproximado de $\mathrm{S} / .81$ millones, atienden al $61 \%$ de la matrícula de estudiantes a nivel nacional.

Por lo que en esta circunstancia se ha realizado la investigación a los impactos de los contenidos del Actividades de supervisión y monitoreo durante el Diplomado en Gestión Escolar y Segunda Especialidad en Gestión Escolar con Liderazgo Pedagógico, que se impartieron a los Directores y Sub Directores del ámbito jurisdiccional de la región Huancavelica.

En esta circunstancia se plasmaron aspectos relacionadas también al contenido del curriculum oculto, que la misma es plasmado en la realidad, como es resaltar de los valores, hábitos, costumbres, sentimientos que los estudiantes de nivel superior adquieren en esta etapa de los trabajos de campo, ahí la importancia que tiene los diferentes trabajos de proyección, trabajos de campo esto en la perspectiva de complementación de los trabajos programados.

Referente a las diferentes zonas trabajadas en la aplicación de los instrumentos de recojo de información en el "Diplomado de Gestión Escolar y Segunda Especialidad en Gestión Escolar con Liderazgo Pedagógico" Sin embargo se presentaron obstáculos, errores, desaciertos y que en el camino fueron superados, tratar acerca de la vigencia del curriculum oculto, fue tomado en cuenta, esto por su trascendencia, importancia en su contenido, que la misma contiene un conjunto de normas, contenidos, metodológicos que orientan al desarrollo de la actividad académica, donde resulta importante catalogar, ordenar el proceso de aprendizaje, enseñanza que son complementados mediante la elaboración, la instrumentación, la aplicación y la correspondiente evaluación a nivel de la región Huancavelica, fueron.

ANGARAES: Vale precisar que se realizó la visita de seguimiento a la Provincia de Angarias, específicamente a Lircay, donde se realizaba el Diplomado de Gestión Escolar con la participación y asistencia de los Directivos de los distritos de la jurisdicción de Angaraes. En esta sede se visitó a la I.E. $\mathrm{N}^{\circ} 36214$ Bellavista - Lircay - Angarias, donde se aplicó los instrumentos de visita inicial - chequeo de Aula y chequeo de la sede de capacitación respectivamente, recopilando datos importantes que contribuyan al mencionado Diplomado, luego retornando a la ciudad de Huancavelica a seguir consolidando la información.

CASTROVIRREYNA: En seguida se realizó el viaje a la provincia de Castrovirreyna, donde se realiza el Diplomado en Gestión Escolar, de este modo se realizó actividades relacionadas con el Diplomado en los diferentes ambientes del Instituto de Educación Superior Tecnológico Público Castrovirreyna, donde se realizó la ejecución del Diplomado Escolar con participación y asistencia de los diferentes participantes Directores y Subdirectores del ámbito territorial de la Provincia de Castrovirreyna, región Huancavelica. 
HUANCAVELICA: Continuando con el desarrollo de la capacitación en Huancavelica, impartido por el Supervisor, facilitador conjuntamente se realizó la visita a la sede del Diplomado, donde la EF venía equipando e implementando la sede del Diplomado, que en esta oportunidad fue el Instituto de Educación Superior Pedagógico Público Huancavelica - IESPPH en donde se desarrolló, constató el proceso de implementación de las aulas donde se realizaba el Diplomado.

De este modo se aplicó los instrumentos de visita Inicial - Lista de Chequeo del Aula, así como la visita Inicial - Lista de chequeo en las diferentes sedes de Capacitación.

En la ciudad de Huancavelica, como en otras ciudades en la década del 80 se presentaron casos de la violencia socio política, por tal motivo emigraron familias enteras a otras ciudades, lugares, pero luego fue nuevamente asentada y ahora es una prospera ciudad con una riqueza cultural e inmaterial que posee, así mismo cuenta con los diferentes servicios, pero que en algunos casos falta de movilidad para trasladarse a las diferentes sedes, distritos y caseríos, como es en este caso para viajar a Angaraes - Lircay.

Para lo cual era necesario poder precisar que existen obstáculos en la vía, puesto que actualmente se encuentra en plena construcción la carretera de Huancavelica a Lircay, que en algunos sectores, tramos se encuentran restringidas, por la cantidad de maquinarias, y obreros que vienen trabajando esta carretera de gran importancia y moderna, demorando de este modo el tiempo de viaje para llegar a esta ciudad importante que es Lircay, donde se realizó el Diplomado en gestión escolar y segunda especialidad en gestión escolar con liderazgo pedagógico, orientado a los Directores y Sub Directores del ámbito jurisdiccional de la región Huancavelica.

Para futuros trabajos en la jurisdicción de la región Huancavelica, es necesario planificar con anticipación la ejecución de trabajos, actividades puesto que existen imprevistos generados por la naturaleza y el medio ambiente, para ello realizar un seguimiento a las metas con la finalidad de establecer avances y alertas.

Dar a conocer a los padres y madres de familia los avances, dificultades y alertas respecto al logro de aprendizajes de los niños y las niñas, con la finalidad de sensibilizarlos y comprometerlos con el logro de resultados.

El monitoreo al avance de los compromisos se puede realizar de forma bimestral o trimestral, según sea el caso. En algunas Jornadas donde podrán participar padres de familia, aliados estratégicos y entre otros, dando a conocer los avances de los compromisos por edad, grado y área curricular, según sea el caso, con el objetivo de reajustar las metas y generar el compromiso de todos los actores de la comunidad, esto en las II.EE. con población estudiantil logrando las metas de aprendizaje.

Referente al ingreso de toda la información acopiada por parte de los facilitadores, especialistas, los diversos datos fueron alimentadas, ingresando a la plataforma electrónica, vale decir los diferentes datos pertinentes a aspectos relacionadas con los contenidos en el Módulo 0, del "Diplomado de Gestión Escolar y Segunda Especialidad en Gestión Escolar con Liderazgo Pedagógico", esta fue de manera práctico, que fueron guiados, basados en el manual, formato de datos, información virtual para el buen uso de los docentes en la mejora de la educación, consecuentemente vale precisar que es necesario resaltar la importancia que tiene los TICs. En esta etapa del trabajo.

En este proceso de cambio, es necesario mencionar que al interior de las diferentes instituciones educativas del nivel primario y secundario, hemos percibido y encontrado realida- 
des diversas, con una diversificación marcada, donde cada vez más se encuentra influenciada por la globalización, a su vez por la tecnología, la modernidad y una economía de mercado que permite mirar hacia afuera, donde los estudiantes de estas localidades buscan emigrar a las grandes ciudades, importantes y en algunos casos al extranjero.

En efecto los diferentes datos que han sido aplicados en las sedes de Huancavelica, Angaraes y Castrovirreyna, fueron ingresados a la plataforma virtual, que en la actualidad se cuenta con la modernidad y globalización que se tiene en los diferentes países, específicamente en el Perú y particularmente en la región Huancavelica, estas se ingresaron todos los datos que se han aplicado mediante los diferentes instrumentos correspondientes al Módulo 0, del Diplomado de Gestión Escolar y Segunda Especialidad en Gestión Escolar.

\section{Discusión}

Desde el "Diplomado en Gestión Escolar y Segunda Especialidad en Gestión Escolar con mención en Liderazgo Pedagógico". Los directivos en la actualidad los Directores y Sub directores se encuentran en la Dirección de las diferentes instituciones educativas del nivel secundario y primario, mediante actividades innovadores en la mejora del plan curricular de estudios acorde a la normatividad vigentes del Minedu, complementados con aspectos y contenidos del curriculum oficial.

Es el centro educativo donde se genera políticas de nivel micro, y se vincula con sus esfuerzos para dar respuesta a los nuevos desafíos, así como para gestar equipos pedagógicos y convocar a los directivos y supervisores con toda su capacidad y creatividad. Implica un cambio radical de hacer escuela, de concebir la acción de los actores educativos con roles de gestión. Se requiere de los equipos de dirección y de supervisión y de apoyo con capacidad de impulsar y promover centros educativos integrados alrededor del desafío de adecuar los procesos de enseñanza a la generación de una experiencia escolar de calidad para poder lograr y elevar los resultados de aprendizajes. Visto desde otra perspectiva, gestión escolar es la capacidad de atender y de renovar el repertorio de problemas a resolver acorde con los desafíos de su tiempo.

Dentro del contexto actual de la educación mediante la puesta en marcha del programa el Minedu, mediante el diplomado capacitó a más de 13,536 mil directores y subdirectores de colegios públicos del país, que mediante diplomado y segunda especialidad se buscaron elevar calidad de la gestión escolar en las instituciones educativas. En efecto fueron capacitados por el Ministerio de Educación, específicamente los directores y sub directores de la región Huancavelica, dentro de ellos Angaraes, Castrovirreyna y Huancavelica respectivamente, con el propósito de que desarrollen competencias y desempeños que les permitan realizar gestiones en beneficio de sus instituciones educativas.

Por cuanto el Minedu a través de la dirección general de calidad de la gestión escolar, viene enmarcando que es la primera vez que se implementa con un programa de formación de directivos a escala, tanto a nivel nacional, regional, nacional. Estas especializaciones se realizan a través las más prestigiosas universidades del país con un programa formativo denominado "Diplomado en Gestión Escolar y Segunda Especialidad en Gestión Escolar con mención en Liderazgo Pedagógico". Los participantes que fueron capacitados en dirección y planificación escolar, mientras que en la Segunda Especialidad recibieron capacitación en gestión curricular y en el monitoreo, acompañamiento y evaluación. 


\section{Conclusión.}

La presente investigación se ha centrado en realizar un trabajo etnográfico, con el propósito de indagar la percepción sobre el "Diplomado en gestión escolar y segunda especialidad en gestión escolar con mención en liderazgo pedagógico, complementados por los contenidos del plan curricular, precisando el cumplimiento de los trabajos de campo, complementado por el método etnográfico, basado en la observación participante dentro de la investigación, que fueron fructíferos los resultados a través de la percepción que demostraron cada uno de los Actividades de supervisión y monitoreo durante el Diplomado en Gestión Escolar y Segunda Especialidad en Gestión Escolar con Liderazgo Pedagógico, que se impartieron a los Directores y Sub Directores del ámbito jurisdiccional de la Región Huancavelica.

Así los contenidos del diplomado motivo a los diferentes directores a aprovechar de este programa y no perder de vista que este esfuerzo que se realiza en beneficio de mejorar la calidad de vida de los docentes, alumnos del territorio peruano, donde los diferentes directores, sub directores se formaron en tres ciclos académicos con certificación progresiva, los dos primeros a través del diplomado en gestión escolar y el tercero conducente al título de segunda especialidad en gestión escolar con liderazgo pedagógico.

En esta etapa del proceso educativo, es necesario resaltar los cambios que se dan al interior de las comunidades, mencionar que al interior de las diferentes instituciones educativas del nivel primario y secundario, hemos percibido y encontrado realidades diversas, con una diversificación marcada, donde cada vez más se encuentra influenciada por la globalización, a su vez por la tecnología, la modernidad y una economía de mercado que permite mirar hacia afuera, donde los estudiantes de estas localidades buscan emigrar a las grandes urbes, ciudades capitales importantes y en algunos casos llegan a emigrar al extranjero, pero que dejan de estudiar es decir lo abandonan sus estudios superiores,

Definitivamente es necesario manifestar que el elemento central para la gestión en las instituciones educativas depende de los Directores y que este programa es parte de la reforma educativa. Donde es notoria esta contribución que fue en beneficio de la formación profesional de los mismos durante dos años, que en la actualidad dichos Directores y Sub directores se encuentran en la Dirección de las Diferentes instituciones educativas del nivel secundario y primario, realizando actividades innovadores en concordancia al plan de estudios que se encuentra acorde a la normatividad vigentes del Minedu, complementados en la práctica por aspectos y contenidos del curriculum oculto que en esta última no hay mayor información bibliográfica.

En este contexto, el diplomado se encuentra dentro del marco del buen desempeño Directivo (Minedu. 2014), que constituye una herramienta del líder pedagógico, con sustento de la literatura sobre gestión escolar, mejora y cambio educativo, instituciones eficaces, calidad educativa, aprendizaje organizativo, donde el diplomado va dirigido a directores y subdirectores de IE públicas de los diferentes niveles, donde participan a través de la evaluación de acceso a cargos directivos, según resolución del Minedu, esto para ejercer a cargos por un periodo de tres años, esto en el marco de la implementación de la Ley Magisterial donde el Director juega un papel importante, orientados a promover y liderar una comunidad educativa de aprendizaje, con interacción con los docentes de su institución educativa, basada en la colaboración mutua, autoevaluación profesional y formación continua, orientada a mejorar la práctica pedagógica y asegurar logros de aprendizaje. 


\section{Referencias.}

Bourdieu, P. (1970). La Reproducción: Elementos para una teoría del sistema de enseñanza. Siglo XXI Editorial.

Cabrera, J. (2006). Juventud y Desarrollo Sustentable en el Perú. Análisis y propuestas a partir de una experiencia de desarrollo local. Ed. Grupo GEA.

Geertz, C. (2007). Interpretación de las Culturas. Barcelona: Gedisa.

Minedu (2014) Gestión de la complejidad y diversidad de la institución educativa.

Minedu (2016) Gestión de la complejidad y diversidad de la institución educativa. Ed. Llankay.

Psyma, (2015) Etnografia. PSYMA.

Restrepo, E. (2016). Etnografia: Técnicas y Éticas. Bogotá: Pontifica Universidad Javeriana. 